CERN-TH/2001-310

UTHET-01-1101

hep-th/0111085

\title{
dS/CFT correspondence on a brane
}

\author{
A. C. Petkou ${ }^{1}$ \\ CERN Theory Division, \\ CH 1211 Geneva 23, \\ Switzerland \\ G. Siopsis ${ }^{2}$ \\ Department of Physics and Astronomy, \\ The University of Tennessee, Knoxville \\ TN 37996 - 1200, USA.
}

\begin{abstract}
We study branes moving in an AdS Schwarzschild black hole background. When the brane tension exceeds a critical value, the induced metric on the brane is of FRW type and asymptotically de Sitter. We discuss the relevance of such configurations to dS/CFT correspondence. When the black hole mass reaches a critical value that depends on the brane tension, the brane interpolates in the infinite past and future between a dS space and a finite space of zero Hubble constant. This corresponds to a cosmological evolution without a Big Bang or a Big Crunch. Moreover, the central charge of the CFT dual to the dS brane enters the Cardy-Verlinde formula that gives the entropy of the thermal CFT dual to the bulk AdS black hole.
\end{abstract}

\footnotetext{
${ }^{1}$ tassos.petkou@cern.ch

2 gsiopsis@utk.edu
} 


\section{Introduction}

The $\mathrm{AdS}_{D+1} / \mathrm{CFT}_{D}$ correspondence [1] is nowadays largely accepted as an established duality, holographical in nature, between a $D+1$-dimensional gravitational theory in the bulk and a D-dimensional CFT in the boundary. A generalisation of holographic ideas leads naturally

to conjecture the existence of a $\mathrm{dS}_{D+1} / \mathrm{CFT}_{D}$ correspondence. In this case, holography takes place on the asymptotic boundaries along the time direction and the dual CFT (CFTs) is (are) Euclidean. Despite some serious puzzles, most notably the unclear nature of a string realization of the dS/CFT correspondence, such a proposal has been recently put forward in a concrete manner and attracted some interest $[2,3]$.

One of the interesting aspects of the conjectured dS/CFT correspondence is the possibility of providing a quantum field theoretical description for fields in de Sitter space. Such a goal is of obvious value in view of the recent experimental observations for the existence of a positive cosmological constant in our universe [4]. Moreover, if the dS/CFT correspondence resembles at all its successful cousin - the AdS/CFT correspondence - then the possibility arises for a quantum field theoretical description of the cosmological evolution [5,6]. Namely, considering that the cosmological evolution in $D$ spacetime dimensions is modelled by a (flat) metric of the FRW form

$$
\mathrm{d} s^{2}=-\mathrm{d} \tau^{2}+\mathcal{R}^{2}(\tau) \mathrm{d} \bar{x}_{D-1}^{2}
$$

such that the Hubble constant

$$
H=\frac{\dot{\mathcal{R}}}{\mathcal{R}} \rightarrow H_{ \pm}, \quad \text { as } \quad \tau \rightarrow \pm \infty
$$

where the dot denotes differentiation with respect to $\tau$, then the cosmological evolution could be viewed as a "holographic" RG flow between the two Euclidean CFTs dual to the asymptotic de Sitter regimes at $\tau= \pm \infty$ of (1). Such an evolution from the infinite past to the infinite future would correspond to a dual RG flow from the IR to the UV.

Motivated by the above, as well as from the puzzling rareness of string/M-theory compactifications to de Sitter space (see however [7]), we consider in this letter branes moving in an $\operatorname{AdS}_{D+1}$ Schwarzschild black hole background. When the brane tension exceeds a critical value we find solutions in which the induced metric on the brane is of the general FRW form and approaches 
de Sitter space in the infinite past and/or future.

An interesting class of solutions are those where the brane radius is always greater than the event horizon of the $\mathrm{AdS}_{D+1}$ black hole and stretches to infinity both at the infinite past and future. In this case the brane metric is asymptotically $\mathrm{dS}_{D}$, with the same Hubble constant in the past and future infinity. The $\mathrm{dS}_{D} / \mathrm{CFT}_{D-1}$ correspondence applied to the asymptotic de Sitter regimes of the above brane metrics implies the existence of dual CFTs whose central charges depend on the asymptotic Hubble constants. In our case, the latter depend generically only on the brane tension, hence it seems that their values have nothing to do with any properties of the bulk $\operatorname{AdS}_{D+1}$ black hole. Phrased differently, the brane excitations induced by the thermal bulk seem to be "orthogonal" to "holographic" de Sitter RG flows. Nevertheless, these brane configurations break down when the mass of the bulk $\mathrm{AdS}_{D+1}$ black holes reaches a critical value that depends on the brane tension. At this critical point, the solution interpolates between a dS space and a space with Hubble constant zero and corresponds to non-singular evolution on the brane (no Big Bang or Big Crunch). It appears therefore that at this critical point the thermodynamics of the $\mathrm{AdS}_{D+1}$ black holes interferes with the $\mathrm{dS}_{D} / \mathrm{CFT}_{D-1}$ correspondence. Indeed, the central charge $c_{D-1}[5]$ of the $\mathrm{CFT}_{D-1}$ is now related to the energy of the black hole, thus entering the Cardy-Verlinde formula [8] that gives the entropy of the thermal $\mathrm{CFT}_{D}$ dual to the black hole $[9]$ as

$$
S=2 \pi \sqrt{\frac{\tilde{C}}{6}\left(A_{D} c_{D-1}-\frac{\tilde{C}}{24}\right)} .
$$

The quantity $\tilde{C}$ is the generalized central charge of the thermal $\mathrm{CFT}_{D}[8]$ and $A_{D}$ is a $D$ -

dependent constant. Since $\tilde{C}$ is the appropriate generalization to more than two dimensions of the number of degrees of freedom of the $\mathrm{CFT}_{D}$ that are thermalized at the critical point $[10,11]$, it is tempting to relate $c_{D-1}$ to the maximum number of degrees of freedom on the brane that can be thermalized by a bulk black hole.

\section{Branes moving in AdS Schwarzschild black holes}

The equations describing the dynamics of codimension one surfaces inside a bulk gravitational theory have been around for a long time [12]. Due to the recent interest in brane world scenaria, 
there exists by now a vast literature on the subject of branes moving in bulk gravity. For our purposes, we take the simple model of a brane moving in the background created by an AdS Schwarzschild black hole. This model has been first studied in the context of AdS/CFT correspondence in [13]. The $D+1$ gravitational action in the presence of a brane is

$$
S=\frac{1}{16 \pi G_{D+1}} \int_{\mathcal{M}}(R-2 \Lambda)+\frac{1}{8 \pi G_{D+1}} \int_{\partial \mathcal{M}} \sqrt{\gamma} \mathcal{K}+\frac{\kappa}{8 \pi G_{D+1}} \int_{\partial \mathcal{M}} \sqrt{\gamma}
$$

where $\mathcal{K}$ is the trace of the the extrinsic curvature $\mathcal{K}_{\mu \nu}=\gamma_{\mu}^{\alpha} \gamma_{\nu}^{\beta} \nabla_{\alpha} \eta_{\beta}$ taken with respect to the induced metric on the brane $\gamma_{\mu \nu}=g_{\mu \nu}-\eta_{\mu} \eta_{\nu}, \kappa$ is related to the brane tension and $\eta_{\mu}$ is the unit normal vector to the brane.

A solution to the bulk equations of motion is given by the AdS Schwarzschild black hole in $D+1$ dimensions

$$
\begin{aligned}
\mathrm{d} s^{2} & =-h(r) \mathrm{d} t^{2}+\frac{\mathrm{d} r^{2}}{h(r)}+r^{2} \mathrm{~d} \Omega_{D-1}^{2} \\
h(r) & =\frac{r^{2}}{L^{2}}+1-\frac{\omega_{D} M}{r^{D-2}}, \quad \omega_{D}=\frac{16 \pi G_{D+1}}{(D-1) V_{D-1}}
\end{aligned}
$$

with $V_{D-1}=2 \pi^{D / 2} / \Gamma(D / 2)$ and $M$ the mass of the black hole. In this normalization, the cosmological constant of $A d S_{D+1}$ is $\Lambda_{D+1}=-D(D-1) / 2 L^{2}$. To derive the equations of motion we use Gaussian normal coordinates in the vicinity of the brane

$$
\mathrm{d} s^{2}=\mathrm{d} \eta^{2}+\gamma_{\mu \nu} \mathrm{d} x^{\mu} \mathrm{d} x^{\nu}
$$

with the position of the brane at $\eta=0$. We then let the coordinates on the brane to be functions of the proper time $\tau$ on the brane as $x^{\mu}=(r(\tau), t(\tau), \bar{\theta})$, where the $\bar{\theta}$ denotes collectively the angular coordinates. The velocity of a static particle (or a piece of stress energy) on the brane is

$$
q^{\mu}=\frac{\mathrm{d} x^{\mu}}{\mathrm{d} \tau}=\left(\frac{\mathrm{d} t}{\mathrm{~d} \tau}, \frac{\mathrm{d} r}{\mathrm{~d} \tau}, \overline{0}\right), \quad q^{\mu} q_{\mu}=-1 .
$$

The second condition above is written as

$$
\frac{1}{h(r)}\left(\frac{\mathrm{d} r}{\mathrm{~d} \tau}\right)^{2}-h(r)\left(\frac{\mathrm{d} t}{\mathrm{~d} \tau}\right)^{2}=-1
$$

which ensures that the induced metric $\gamma_{\mu \nu}$ on the brane is of the FRW type

$$
\mathrm{d} s_{\text {brane }}^{2}=-\mathrm{d} \tau^{2}+r^{2}(\tau) \mathrm{d} \Omega_{D-1}^{2} .
$$


In the coordinates $(7)$ the normal vector to the brane (i.e. at $\eta=0$ ), pointing in the direction of increasing $r$, is simply $\eta_{\mu}=(1,0, \overline{0})$. To derive the equations of motion, however, we need the normal vector in the vicinity of the brane which is determined by

$$
\eta^{\mu} q_{\mu}=0, \quad \eta^{\mu} \eta_{\mu}=1
$$

These are easily solved to give

$$
\eta_{\mu}=(-\dot{r}, \dot{t}, \overline{0}), \quad \dot{t}=\frac{\mathrm{d} t}{\mathrm{~d} \tau}, \quad \dot{r}=\frac{\mathrm{d} r}{\mathrm{~d} \tau}
$$

Now, the equation of motion of the brane is given by the Israel junction conditions [12] which, assuming that $\eta_{\mu}$ points in the direction of increasing $r$, read

$$
\mathcal{K}_{\mu \nu}=T_{\mu \nu}-T_{\rho}^{\rho} \frac{1}{D-1} \gamma_{\mu \nu}
$$

$T_{\mu \nu}$ is the energy momentum tensor on the brane which here describes just its vacuum energy and is given by

$$
T_{\mu \nu}=-\kappa \gamma_{\mu \nu}
$$

Then (13) becomes

$$
\mathcal{K}_{\mu \nu}=\frac{\kappa}{D-1} \gamma_{\mu \nu}
$$

To proceed we calculate the extrinsic curvature on the brane noticing the relation

$$
\partial_{\eta}=\eta^{\mu} \frac{\partial}{\partial x^{\mu}}
$$

It suffices to consider just one of the angular components of $\mathcal{K}_{\mu \nu}$, e.g., the $\mathcal{K}_{\theta \theta}$ component in the coordinate system $(7)$ is

$$
\mathcal{K}_{\theta \theta}=-\Gamma_{\theta \theta}^{\eta} \eta_{\eta}=\frac{1}{2} \eta^{\mu} \partial_{\mu} r^{2}=r h(r) \dot{t}
$$

Then (15) yields

$$
\dot{t}=\frac{\kappa r}{(D-1) h(r)} .
$$

The gravitational constant of the induced metric can be easily calculated [14] as

$$
\Lambda_{D}=\frac{D-2}{D}\left(\Lambda_{D+1}+\frac{D}{2(D-1)} \kappa^{2}\right) \equiv \frac{D-2}{2(D-1)}\left(\kappa^{2}-\kappa_{c}^{2}\right)
$$


where (19) also defines the critical tension $\kappa_{c}$. Finally, using (9) we obtain from (18)

$$
H \equiv\left(\frac{\dot{r}}{r}\right)^{2}=\frac{2 \Lambda_{D}}{(D-2)(D-1)}-\frac{1}{r^{2}}+\frac{\omega_{D} M}{r^{D}} .
$$

This equation describes the cosmological evolution for the brane universe in the AdS Schwarzschild black hole background. Similar equations have been studied in various braneworld related contexts in a number of references [15].

\section{Asymptotically dS metrics on branes}

\subsection{The $D=4$ case}

We start our analysis of (20) by considering in detail the case $D=4$ when we have

$$
\dot{r}^{2}=\frac{\Lambda_{4}}{3} r^{2}-1+\frac{\omega_{4} M}{r^{2}}
$$

The solution when the brane cosmological constant is zero corresponds to fine tuning the brane tension to $\kappa=\kappa_{c}$ and has been studied in detail in [8]. For $0<\kappa<\kappa_{c}$ we obtain negative cosmological constant on the brane. ${ }^{3}$ In this case we denote $H_{1}^{2}=-\Lambda_{4} / 3>0$ and notice that for $M=0$, which corresponds to the bulk space being pure AdS, there exists no solution. For $M>0$, we obtain in terms of $x=r^{2}$

$$
\dot{x}^{2}=-4 H_{1}^{2}\left(x-\mathrm{x}_{-}\right)\left(x-\mathrm{x}_{+}\right), \quad \mathrm{x}_{ \pm}=-\frac{1}{2 H_{1}^{2}}\left[1 \mp \sqrt{1+4 \omega_{4} M H_{1}^{2}}\right] .
$$

Only $\mathrm{x}_{+}$is positive, thus we have a bounded motion in the interval $x(\tau)=r^{2}(\tau) \in\left(0, \mathrm{x}_{+}\right]$. Then, the solution is

$$
x(\tau) \equiv r^{2}(\tau)=\frac{1}{2 H_{1}^{2}}\left[\sqrt{1+4 \omega_{4} M H_{1}^{2}} \cos \left(2 H_{1} \tau\right)-1\right],
$$

which describes an oscillating universe.

For $\kappa>\kappa_{c}$ we obtain a positive cosmological constant on the brane. Denoting $H_{2}^{2}=\Lambda_{4} / 3$ we obtain from $(21)$

$$
\frac{1}{4} \dot{x}^{2}=H_{2}^{2} x^{2}-x+\omega_{4} M=H_{2}^{2}\left(x-x_{-}\right)\left(x-x_{+}\right),
$$

\footnotetext{
${ }^{3}$ We do not consider here the unclear case of having negative tension branes.
} 
where

$$
x_{ \pm}=\frac{1}{2 H_{2}^{2}}\left(1 \pm \sqrt{1-4 \omega_{4} M H_{2}^{2}}\right) .
$$

We begin with the extremal case $M=0$, i.e., when the bulk is pure AdS. The solution is then

$$
r(\tau)=\frac{1}{H_{2}} \cosh \left(H_{2} \tau\right)
$$

which in view of (10) describes a de Sitter universe. This case has been studied from different perspectives, e.g., in $[15,16]$.

If $4 \omega_{4} M H^{2}<1$, both roots (25) are positive. In this case there are two possible orbits, one in the interval $\left(0, x_{-}\right]$and another in the interval $\left[x_{+}, \infty\right)$. Since one easily verifies that the black hole horizon distance satisfies $r_{H}<x_{-}, x_{+}$, in the first orbit above the brane crosses the horizon at finite proper time, while in the second orbit the brane never crosses the horizon. Eq. (24) can be solved exactly. After some algebra, we find that for $x \leq x_{-}$,

$$
r^{2}(\tau)=x(\tau)=\frac{1}{2 H_{2}^{2}}\left(1-\sqrt{1-4 \omega_{4} M H_{2}^{2}} \cosh \left(2 H_{2} \tau\right)\right),
$$

whereas for $x \geq x_{+}$,

$$
r^{2}(\tau)=x(\tau)=\frac{1}{2 H_{2}^{2}}\left(1+\sqrt{1-4 \omega_{4} M H_{2}^{2}} \cosh \left(2 H_{2} \tau\right)\right) .
$$

When $4 \omega_{4} M H_{2}^{2}>1$, we obtain the solution

$$
r^{2}(\tau)=x(\tau)=\frac{1}{2 H_{2}^{2}}\left(1+\sqrt{4 \omega_{4} M H_{2}^{2}-1} \sinh \left( \pm 2 H_{2} \tau\right)\right) .
$$

Finally, for $4 \omega_{4} M H_{2}^{2}=1$ we obtain the solutions

$$
r^{2}(\tau)=x(\tau)=\frac{1}{2 H_{2}^{2}}\left(1 \pm e^{ \pm 2 H_{2} \tau}\right)
$$

At this critical point there are two possible orbits one of which interpolates between a dS space of Hubble constant $H_{2}$ and a space of $H=0$. This corresponds to a non-singular evolution (without a Big Bang or a Big Crunch, depending on the sign choice for $H_{2}$ ). The various orbits are shown in Fig. 1.

From our perspective the most interesting solution is (28). In this case the brane metric (10) is asymptotically de Sitter both at the past and future infinity and furthermore it never crosses the 


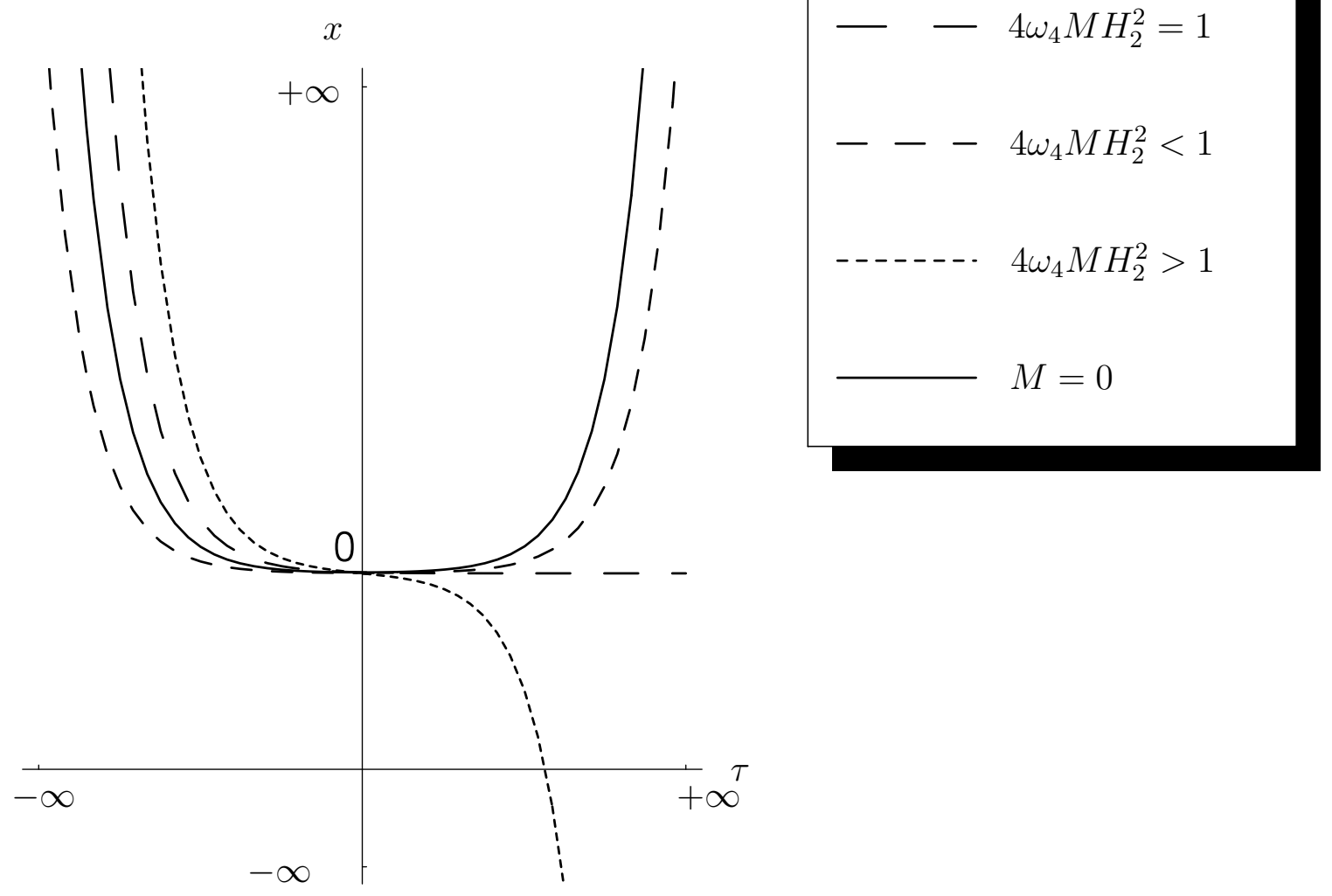

Figure 1: The various brane orbits. For $4 \omega_{4} M H_{2}^{2}<1$ we depict only the $x>x_{+}$orbit. For $4 \omega_{4} M H_{2}^{2}>1$ the brane ceases to exist $(x<0)$ at finite proper time.

black hole horizon. The brane observer sees a cosmological evolution during which the universe starts very big at an inflationary de Sitter era, contracts to a minimum size and then expands again to reach a de Sitter era in the infinite future. Both the past and the future asymptotically de Sitter regimes have the same Hubble constant $H_{2}$. In this sense, our brane universe is far from describing the observed cosmological evolution where the initial and final values of the Hubble constant differ by $\sim 10^{52}$ orders of magnitude. Nevertheless, our solution provides a simple model to test the recent ideas of $\mathrm{dS}_{4} / \mathrm{CFT}_{3}$ correspondence in the context of the cosmological evolution $[5,6]$. According to such ideas, the asymptotically de Sitter regimes in the infinite past and future are respectively dual to a three-dimensional CFT on the sphere $S^{3}$ with radius $r$. The 
central charge of this dual $\mathrm{CFT}_{3}$ is given by [5]

$$
c_{3}=\frac{a_{3}}{H_{2}^{2} G_{4}}
$$

where $G_{4}$ is the gravitational constant on the brane. The parameter $a_{3}$ is a constant. ${ }^{4}$ For general $0 \leq 4 \omega_{4} M H_{2}^{2}<1$, we see from (19) that $H_{2}$ is independent of the black hole mass, hence the central charge of the dual $\mathrm{CFT}_{3}$ depends essentially only on the brane tension $\kappa$. Therefore, although the presence of a black hole in the bulk distorts the orbit and from purely de Sitter makes it only asymptotically de Sitter, there does not seem to be any interaction of the black hole degrees of freedom with the degrees of freedom on the brane that are relevant to the boundary $\mathrm{CFT}_{3}$, i.e. this particular brane evolution is "orthogonal" to the RG flow of the $\mathrm{CFT}_{3}$. However, when the black hole mass reaches the critical value

$$
M=M_{c}=\frac{1}{4 \omega_{4} H_{2}^{2}},
$$

then there seems to be an interference between the degrees of freedom of the black hole and those of the $\mathrm{CFT}_{3}$. In this case, starting, for example, the evolution from a de Sitter regime in the infinite past, the brane contracts and never re-expands again reaching a spatially homogeneous space with zero Hubble constant in the infinite future. From the point of view of the $\mathrm{CFT}_{3}$ such an evolution corresponds to a RG flow from an IR regime, where a finite number of degrees of freedom are coupled, to an UV regime where the coupled degrees of freedom are infinite.

On the other hand, when $M>M_{c}$ we see from (29) that $r^{2}(\tau)$ becomes negative at finite proper time which indicates that the brane configuration ceases to exist. In that sense, $c_{3}$ in (Eq. (31)) provides an upper bound on the energy of black holes that can support brane configurations stretching from the infinite past to the infinite future. Indeed, using the equation $h\left(r_{H}\right)=0$ that gives the horizon distance $r_{H}$ of the black hole, the condition $M \leq M_{c}$ translates to

$$
c_{3}=\frac{a_{3}}{H_{2}^{2} G_{4}} \geq \frac{a_{3}}{G_{4}} 4 r_{H}^{2}\left(1+\frac{r_{H}^{2}}{L^{2}}\right) .
$$

\footnotetext{
${ }^{4}$ The parameter $a_{3}$ can presumably be calculated from the correlators of the energy momentum tensor of the $\mathrm{CFT}_{3}$. The latter can be calculated from graviton correlators in $\mathrm{dS}_{4}$. Notice however, that the cosmological implementation above of the dS/CFT correspondence requires the definition of a central charge for an odd-dimensional CFT. Contrary to common misconceptions in the current literature, the notion of a quantity measuring the degrees of freedom coupled to a critical point is not necessarily connected to the conformal anomaly and exists in any dimension. For various 3-dimensional examples see e.g. [17].
} 
The equality in (33) associates a "critical" $\mathrm{CFT}_{3}$ to an $\mathrm{AdS}_{5}$ Schwarzschild black hole of radius $L$ and horizon distance $r_{H}$. Apparently, the bulk AdS black hole does know something about the "critical" theory whose central charge is given by (31). Using the standard brane-world relation

$$
G_{D+1}=\frac{L}{D-2} G_{D}
$$

we obtain from $(33)$

$$
c_{3}=a_{3} \frac{16}{3 \pi} E_{c r i t} L \gtrsim a_{3} \frac{16}{3 \pi} E L
$$

where $E$ is the energy of the black hole that supports a brane cosmology and which is identified with the total thermal energy of the dual $\mathrm{CFT}_{4} . E_{\text {crit }}$ is the energy of the black hole at the critical point $M=M_{c}$. At this critical point, using the definition of the Casimir entropy $S_{C}$

$$
S_{C}=S \frac{L}{r_{H}} \equiv \frac{\pi}{6} \tilde{C},
$$

where $S$ is the entropy and $\tilde{C}$ the generalized central charge $[8,10,11]$, we can write the entropy of the black hole as

$$
S=2 \pi \sqrt{\frac{\tilde{C}}{6}\left(\frac{\pi}{16 a_{3}} c_{3}-\frac{\tilde{C}}{24}\right)} .
$$

Thus, the central charge of the $\mathrm{CFT}_{3}$ enters the Cardy-Verlinde formula for the entropy of the thermal $\mathrm{CFT}_{4}$ at the critical point $M=M_{c}$.

\subsection{Generalization to $D>4$}

For $D>4$ we consider only the case with a positive cosmological constant on the brane when Eq. (21) generalizes to

$$
\dot{r}^{2}=H_{2}^{2} r^{2}-1+\frac{\omega_{D} M}{r^{D-2}}
$$

with $H_{2}^{2}=\frac{2 \Lambda_{D}}{(D-2)(D-1)}$. This cannot be solved exactly, but we may demonstrate the qualitative features we encountered in $D=4$. To this end, first rewrite the equation as

$$
r^{D-2} \dot{r}^{2}+f(r)=0 \quad, \quad f(r)=-H_{2}^{2} r^{D}+r^{D-2}-\omega_{D} M
$$

The potential $f(r)$ has a maximum at $r_{\max }$, where $f^{\prime}\left(r_{\max }\right)=0$, i.e.,

$$
r_{\text {max }}^{2}=\frac{(D-2)}{D H_{2}^{2}} \text {. }
$$


As in $D=4$, we have three cases:

If $f\left(r_{\max }\right)>0$, then there are two orbits, one of which approaches $r \rightarrow \infty$ in the infinite past and future. We obtain $\dot{r}^{2} \approx H_{2}^{2} r^{2}$, therefore,

$$
r \sim e^{ \pm H_{2} \tau}
$$

and the brane interpolates between dS spaces of Hubble constant $\mathrm{H}_{2}$.

If $f\left(r_{\max }\right)<0$, then the brane starts from a dS space of Hubble constant $H$ in the infinite past and falls into the black hole singularity.

At the critical point $f\left(r_{\max }\right)=0$, the mass of the black hole becomes $M=M_{c}$, where $M_{c}$ satisfies

$$
\frac{1}{H_{2}^{D-2}}=\frac{D-2}{2}\left(\frac{D}{D-2}\right)^{\frac{D}{2}} \omega_{D} M_{c} .
$$

The brane interpolates between a dS space of Hubble constant $H_{2}$ in the infinite future and a finite space of radius $r=r_{\max }$ in the infinite past. There is also another orbit that approaches the latter point starting from the singularity $r=0$. To see the behaviour near $r \rightarrow r_{\max }$, expand

$$
f(r)=\frac{1}{2} f^{\prime \prime}\left(r_{\max }\right)\left(r-r_{\max }\right)^{2}+\ldots=(D-2) r_{\max }^{D-4}\left(r-r_{\max }\right)^{2}+\ldots
$$

Therefore,

$$
\dot{r}^{2}=-\frac{f(r)}{r^{D-2}} \approx-\frac{(D-2)}{r_{\max }^{2}}\left(r-r_{\max }\right)^{2}=-D H^{2}\left(r-r_{\max }\right)^{2},
$$

whose solution is

$$
r-r_{\max } \sim e^{ \pm \sqrt{D} H}
$$

In this case, using (42) and the equation for the black hole horizon, the central charge of the $\mathrm{CFT}_{D-1}$ dual to the asymptotic $\mathrm{dS}_{D}$ regime is

$$
c_{D-1}=\frac{a_{D-1}}{H^{D-2} G_{D}}=\frac{D-2}{2}\left(\frac{D}{D-2}\right)^{\frac{D}{2}} r_{H}^{D-2}\left(1+\frac{r_{H}^{2}}{L^{2}}\right) \frac{a_{D-1}}{G_{D}},
$$

which using (34) and the formula that gives the energy of the bulk black hole [9] can be written as

$$
c_{D-1} \frac{8 \pi a_{D-1}}{V_{D-1}(D-1)}\left(\frac{D}{D-2}\right)^{\frac{D}{2}} E_{c r i t} L .
$$

The $D$-dependent parameter $a_{D-1}$ is a constant (see Footnote 1). Away from the critical point, the condition for the existence of asymptotic dS spaces is $E<E_{c r i t}$. Thus $c_{D-1}$ provides an 
upper bound for the energy of the black hole which generalizes (35). Moreover, the CardyVerlinde formula for the entropy of the thermal $\mathrm{CFT}_{D}$ is written at the critical point $M=M_{c}$ as

$$
S=2 \pi \sqrt{\frac{\tilde{C}}{6}\left(\frac{V_{D-1}}{8 \pi}\left(\frac{D-2}{D}\right)^{\frac{D}{2}} \frac{c_{D-1}}{a_{D-1}}-\frac{\tilde{C}}{24}\right)}
$$

generalising (37).

\section{Discussion of the results}

The recent experimental observations pointing towards the existence of a positive cosmological constant in our universe has brought up the question of studying quantum field theory in de Sitter space. In that direction, the conjectured dS/CFT correspondence is an interesting development. A further possibility arising in this context is the description of the cosmological evolution via a RG flow between Euclidean CFTs dual to the asymptotic dS regimes of the infinite past and future. With the above in mind, it seems natural to look for realizations of the dS/CFT correspondence in spaces embedded into higher dimensional bulk manifolds. In this way one might hope to use the full range of the existing string theory and supergravity solutions for the bulk to study the dS/CFT correspondence realized on branes.

In this letter, we initiate such an approach to dS/CFT correspondence by studying the simple model of a brane moving in the vicinity of an AdS Schwarzschild black hole. When the tension is greater than a certain critical value, the metric on the brane is of a generic FRW form which is asymptotically de Sitter in the infinite past and/or future. We argue that such a simplified configuration is a good starting point to study properties of the dS/CFT correspondence. An interesting question is whether the bulk theory is at all related to the properties of the CFT(s) dual to the asymptotic dS regimes. Such a possibility would then allow one to use information from the well established AdS/CFT correspondence to study the dS/CFT correspondence.

Our analysis shows that although a black hole in the bulk distorts the orbit of a brane moving into it, in general it does not seem to interfere with the CFTs dual to the asymptotic dS regimes on the brane. However, for a critical value of the black hole mass the asymptotic properties of the brane metric are dramatically altered and the brane interpolates between a dS space in the 
infinite future/past and a space of zero Hubble constant in the infinite past/future. This is a non-singular evolution (without a Big Bang/Big Crunch). Such a critical point defines an upper bound on the energy of a Schwarzschild AdS black hole that can support an asymptotically de Sitter brane cosmology. We view this as an indication that the black hole knows something about the CFT dual to the $\mathrm{dS}$ regimes. This is further supported by observing that at the critical point the central charge of the $\mathrm{CFT}_{D-1}$ enters the Cardy-Verlinde entropy formula (3) of the thermal $\mathrm{CFT}_{D}$ dual to the black hole. Such an observation indicates that the central charge of the $\mathrm{CFT}_{D-1}$ may be related to a quantity of the thermal $\mathrm{CFT}_{D}$. From the Cardy-Verlinde formula (3) one is tempted to relate $c_{D-1}$ to the maximum number of degrees of freedom on the brane that can be thermalized by the bulk black hole, for temperatures above the Hawking-Page transition point [9].

Our arguments above require the existence of a well-defined central charge for a $D$-dimensional CFT. In the context of the dS/CFT correspondence, this means a general formula for the constant $a_{D-1}$ in (46). Such a formula is, of course, not known for $D>2$. In even dimensions the central charges are usually related to conformal anomalies and this makes their study relatively simple. Nevertheless, central charges have been defined in odd dimensions [17] and could be used to study properties of the dS/CFT correspondence in the cosmological evolution setting.

The simple model presented here shows the possibility of studying properties of the dS/CFT correspondence in embedded spaces (i.e. branes), using results of the AdS/CFT correspondence. One could extend our result to asymptotically flat brane cosmologies in which case we expect that one should use the Cardy-Verlinde entropy formula for black holes with flat horizons discussed in [11]. Notice that at the critical point the evolution on the brane leads to an infinite ratio of the Hubble constants in the infinite future and infinite past, respectively. It would be interesting to perturb this symmetric state and obtain a large but finite ratio of Hubble constants as observed in our universe. In that sense, a natural extension of our studies would be to consider more complicated black hole solutions of supergravity and study the influence of the bulk fields on possible asymptotic dS regimes on the brane. Introducing explicitly thermal conformal fields on the brane would also be a possible way forward. In any case, the idea of viewing the cosmological expansion as a RG flow is by itself interesting and worths further study. 


\section{Acknowledgements}

A. C. Petkou would like to acknowledge discussions with Y. Oz and J. Pawelczyk.

\section{References}

[1] J. Maldacena, "The large $N$ limit of superconformal field theories and supergravity", Adv. Theor. Math. Phys. 2 (1998) 231, hep-th/9711200;

G.G. Gubser, I.R. Klebanov and A.M. Polyakov, "Gauge theory correlators from noncritical string theory", Phys. Lett. B428 (1998) 105, hep-th/9802109;

E. Witten, "Anti-de Sitter space and holography", Adv. Theor. Math. Phys. 2 (1998) 253, hep-th/9802150.

[2] A. Strominger, " The dS/CFT correspondence", hep-th/0106113.

[3] D. Klemm, "Some aspects of the de Sitter/CFT correspondence", hep-th/0106247.

[4] S. Perlmutter et. al. [Supernova Cosmology Project Collaboration], "Measurements of Omega and Lambda from 42 high-redshift supernovae", Astrophys. J. 517, (1999) 565.

[5] A. Strominger, "Inflation and the dS/CFT correspondence", hep-th/0110087.

[6] V. Balasubramanian, J. de Boer and D. Minic, "Mass, entropy and holography in asymptotically de Sitter spaces", hep-th/0110108.

[7] C. M. Hull, "De Sitter Space in supergravity and M theory", hep-th/0109213

[8] E. Verlinde, "On the holographic principle in a radiation dominated universe," hepth/0008140.

[9] E. Witten, "Anti-de Sitter space, thermal phase transition and confinement in gauge theories", Adv. Theor. Math. Phys. 2 (1998) 505, hep-th/9803131.

[10] D. Klemm, A. C. Petkou, G. Siopsis, "Entropy bounds, monotonicity properties and scaling in CFTs", Nucl.Phys. B601 (2001) 380, hep-th/ 0101076. 
[11] D. Klemm, A. C. Petkou, G. Siopsis, D. Zanon, "Universality and a generalized C-function in CFTs with AdS duals", hep-th/0104141, to appear in NPB.

[12] S. K. Blau, E. I. Guendelman and A. H. Guth, The dynamics of false vacuum bubbles", Phys. Rev. D35 (1987) 1747.

[13] I. Savonije and E. Verlinde, "CFT and entropy on the brane," Phys.Lett. B507 (2001) 305, hep-th/0102042.

[14] T. Shiromizu, K. Maeda and M. Sasaki, "The Einstein equations on the 3-Brane world", Phys.Rev. D62 (2000) 024012, gr-qc/9910076.

[15] N, Kaloper, "Bent Domain worlds as braneworlds", Phys.Rev. D60 (1999) 123506, hepth/9905210.

P. Kraus, "Dynamics of Anti-de Sitter domain walls", JHEP 9912 (1999) 11, hepth/9910149.

T. Shiromizu and D. Ida, "Anti-de Sitter, no hair AdS/CFT and the brane world", Phys.Rev. D64 (2001) 044015, hep-th/0102035.

S. Nojiri and S. D. Odintsov, "AdS/CFT and quantum corrected brnae entropy", hepth/0103078. "Conformal anomaly from dS/CFT correspondence", hep-th/0106191. "Quantum cosmology, inflationary brane-world creation and dS/CFT correspondence", hepth/0107134.

L. Anchordoqui, C. Nunez and K. Olsen, Quantum cosmology and AdS/CFT", JHEP 0010 (2000) 050, hep-th/0007064,

L. Anchordoqui, J. Edelstein, C. Nunez, S. P. Bergliaffa, M. Schvellinger, M. Trobo and F. Zyserman, "Brane worlds, string cosmology, and AdS/CFT", Phys.Rev. D64 (2001) 084027, hep-th/0106127.

R. Gregory and A. Padilla, "Brane world instantons", hep-th/0107108.

[16] O. DeWolfe, D. Z. Freedman, S. S. Gubser and A. Karch, "Modelling the fifth dimension with scalars and gravity", Phys.Rev.D62 (2000) 046008, hep-th/9909134. 
[17] A. C. Petkou, " $C(T)$ and $C(J)$ up to next-to-lading order in $1 / N$ in the conformally invariant $O(N)$ model for $2<D<4$, Phys.Lett. B359 (1995) 101, hep-th/9506116;

A. C. Petkou and G. Siopsis, "Renormalization group flow and thermodynamics of conformal field theories",JHEP 0002 (2000) 002, hep-th/9906085. 